\title{
Impact of Adherence to a Full Course of Intermittent Preventive Treatment of Malaria in Pregnancy on Pregnancy Outcome in Muyuka Health District: A Cross-Sectional Study
}

Aldof Tah Yoah ${ }^{1,2^{*}}$, Jerome Fru-Cho ${ }^{2}$, Emmanuel Kah ${ }^{1,2}$, Ernest Njukang ${ }^{1,2}$, Franklin Sevidzem Wirsiy $^{1,2}$, Christian Tetteh Duamor ${ }^{2,3}$ and Theresa Nkuo-Akenji ${ }^{2}$

${ }^{1}$ Department of Public Health and Hygiene, University of Buea, Buea 63, Cameroon

${ }^{2}$ Department of Microbiology and Parasitology, University of Buea, Buea 63, Cameroon

${ }^{3}$ Accra School of Hygiene, Ministry of Health, Korle-Bu, Accra, Ghana

*Corresponding author: Aldof Tah Yoah, Department of Public Health and Hygiene, Department of Microbiology and Parasitology, University of Buea, Buea 63, Cameroon, E-mail:yataldof85@yahoo.ca

\begin{abstract}
Background: Intermittent preventive treatment of malaria in pregnancy with sulphadoxine-pyrimethamine (IPTp-SP) is a key strategy for the control of malaria in pregnancy in Sub-Saharan Africa.

Objectives: In Cameroon, the revised IPTp-SP policy requiring that pregnant women take at least three doses of SP was adopted in 2013 but the adherence and its association with pregnancy outcomes remains largely unknown in most rural parts of Cameroon.

Methods: To address these issues, the present study was designed as a cross-sectional community-based survey in which a structured questionnaire and chart review (medical record reviews) were used to collect relevant information from 430 mothers of children below one year of age residing in 14 clusters selected randomly from the Muyuka Health District. Data were entered and analysed using Epi Info 7.

Results: The adherence to a full course of IPTp-SP was $32.09 \%$. Women who adhered to a full course of IPTp-SP had higher mean birth weight infants and mean gestation age at delivery compared to their counterparts who took a partial course (mean birth weight difference $=263 \mathrm{~g}, \mathrm{p}$ $<0.001$; mean gestation age at delivery difference $=0.69$ weeks, $p=0.003$ ). Adherence to a full course of IPTp-SP significantly reduced the odds of malaria in pregnancy (AOR $=0.30, p<0.001$ ) but not that of low birth weight and preterm birth. The effectiveness of a full course of IPTp-SP in preventing clinical malaria in pregnancy was $70 \%$.

Conclusion: Generally, the adherence to full course of IPTp-SP was low. Adherence to full course of IPTp improved birth outcomes. Data generated from the study should be used by health planners to improve on the quality of maternal and infant health care services.
\end{abstract}

\section{Keywords}

Adherence, Full course IPTp-SP, Pregnancy outcomes

\begin{abstract}
Abbreviations
ANC: Antenatal Care; AOR: Adjusted Odds Ratio; DHSMICS: Demographic and Health Survey and Multiple Indicator Cluster Survey; IPTp-SP: Intermittent Preventive Treatment in Pregnancy using Sulphadoxine-Pyrimethamine; FHSIRB: Faculty of Health Sciences Institutional Review Board; ITN: Insecticide-Treated Nets; LBW: Low Birth Weight; MIP: Malaria in Pregnancy; NMCP: National Malaria Control Programme; PPS: Probability Proportionate to Size; PTD: Pre-term delivery; SP: Sulphadoxine-Pyrimethamine; WHO: World Health Organization
\end{abstract}

\section{Background}

Malaria is an acute febrile disease caused by infection of the red blood cells with intracellular protozoan parasites of the genus Plasmodium. The parasites are inoculated into the human host by feeding female Anopheles mosquitoes [1]. Malaria infection during pregnancy presents significant risks for the pregnant woman, the developing foetus and the newborn infant. The negative consequences associated with malaria in pregnancy include severe malaria, severe anaemia, pre-term delivery, maternal death, and placental malaria [2]. Placental malaria is linked to intrauterine growth restriction, stillbirth, and delivery of low birth weight (LBW) infants. Pre-term delivery and LBW are the risk factors for neo-

Citation: Yoah AT, Fru-Cho J, Kah E, Njukang E, Wirsiy FS, et al. (2018) Impact of Adherence to a Full Course of Intermittent Preventive Treatment of Malaria in Pregnancy on Pregnancy Outcome in Muyuka Health District: A Cross-Sectional Study. Int Arch Public Health Community Med 2:008 Received: April 25, 2017; Accepted: May 17, 2018; Published: May 19, 2018

Copyright: (C) 2018 Yoah AT, et al. This is an open-access article distributed under the terms of the Creative Commons Attribution License, which permits unrestricted use, distribution, and reproduction in any medium, provided the original author and source are credited. 
natal and infant deaths [3,4]. In 2013, an estimated 30 million pregnant women were at risk of getting malaria worldwide. There were an estimated 584,000 malaria deaths worldwide. Ninety percent of global malaria deaths occurred in sub-Saharan Africa [2]. In Cameroon, the morbidity due to severe malaria in pregnant women was $52 \%$ while mortality stood at $14 \%$ [5].

In high-malaria transmission zones like sub-Saharan Africa, women are semi-immune, and most malaria infections in pregnancy are asymptomatic. Thus, a preventive approach is appropriate to handle malaria in pregnancy in the said areas. The World Health Organisation (WHO) recommends a three-pronged intervention for the prevention and control of Malaria in Pregnancy (MIP) in areas of stable transmission of malaria namely Intermittent Preventive Treatment in pregnancy using sulphadoxine-pyrimethamine (IPTp-SP), use of insecticide treated nets (ITN) and ensuring effective case management of malaria illnesses and anaemia [6]. In Cameroon, the National Malaria Control Program also insists on this three-pronged intervention for the prevention and control of MIP. In 2012, a Cameroon survey suggested that $59.7 \%$ of households possessed at least one net while only $42.6 \%$ of households slept under these nets [7]. Indoor Residual Spray is not a policy option in Cameroon.

In 2013, the WHO revised its IPTp guideline which continues to recommend the use of Sulphadoxine-Pyrimethamine (SP), but at more frequent dosing due to a reduction in SP half-life associated with parasite resistance [8]. Following the implementation of this revised IPTp-SP protocol, only $17 \%$ of the pregnant women received a full course (three or more doses of SP) of IPTp worldwide. In 2014, a Cameroon household survey indicated that $26 \%$ of pregnant women received a full course of IPTp-SP [9]. All these studies revealed low uptake of IPTp-SP by pregnant women. This increase in the frequency of SP dosing is challenging in sub-Saharan Africa characterised by low IPTp-SP uptake.

Many studies in the area of IPTp-SP uptake have focused on the barriers and pregnancy outcomes associated with the uptake of optimal (two) doses of SP [10]. Following the revision of the IPTp-SP protocol in 2013, little is known on the level of uptake, predictors and pregnancy outcomes associated with the revised recommendation ( $\geq$ three doses of SP) in most of the malaria endemic areas of Sub-Saharan Africa. Thus, the aim of this study was to assess the adherence and pregnancy outcomes associated with the uptake of a full course of IPTp-SP.

\section{Materials and Methods}

\section{Study design}

The study was a cross-sectional community-based survey that took place in the months of April to June 2015.

\section{Study area and setting}

The study took place in the Muyuka Health District located on the leeward side of Mount Cameroon. Muyuka Health District is one of the eighteen health districts of the South West Region of Cameroon. It is situated in Fako Division. The Muyuka Health District as of 2014 had an estimated population of 100,759 inhabitants (3829 women with children less than one year i.e. $3.8 \%$ of the total population) distributed into 5 health areas; Bafia, Meanja, Ekona, Malende and Muyuka. Each health area is divided into administrative units called "quarters". Twenty health units; public, confessional, and private are found in the Muyuka Health District. Of this number, fifteen have been identified for effectively carrying out ANC services and deliveries. This health district is in a malaria-endemic zone where the transmission of malaria is stable. The morbidity of malaria is $28 \%$ for the general population, with $45 \%$ in children less than five years and $17 \%$ in pregnant women [5]. Severe malaria represents $56 \%$ and $52 \%$ of hospitalizations respectively for children below five years and pregnant women respectively. The mortality due to malaria in the entire population is $8 \%$, with $21 \%$ and $14 \%$ in children below five years and pregnant women respectively [5].

\section{Study sites}

We collected data from the Muyuka Health District. The number of participants involved in the study per health area was determined by sampling proportionate to the size of the population.

\section{Target population}

The target population was made up of mothers of children less than one-year-old.

\section{Inclusion criteria}

Any mother of a child less than one-year-old residing in any of the selected health areas in Muyuka Health District for more than nine months or who attended ANC in any of the health facilities.

\section{Exclusion criteria}

Any mother of any woman who gave birth to twins, triplets or quadruplets was excluded birth weight analysis.

\section{Sample size}

The following formula was used to compute the sample size [11]:

$$
\mathbf{n}=\frac{Z^{2} * P *(1-P)}{d^{2}}
$$

$\mathrm{n}=$ Number of participants needed for the study

$Z=1.96$ for $95 \%$ confidence interval

$\mathrm{P}=$ Proportion of IPT1 in community survey in Cameroon (47\%) [5] 
$d=$ Precision $(5 \%)$

$\mathrm{n}=\frac{1.96^{2} * 0.47 *(1-0.47)}{0.05}=382$ (minimum number of

participants in the study).

The sample size was then increased to 430 participants (approximately 10\%) to account for anticipated non-respondents and poorly filled questionnaires.

\section{Sampling Method}

Multistage cluster sampling method was used to select participants from the study area. Three of the five health areas that make up the MHD namely Bafia, Malende and Muyuka were randomly selected. Based on the sample size, 14 clusters ('quarters') were randomly selected from the 47 clusters in our study area. Approximately 30 nursing mothers were selected per clusters (quarter: Has a population of approximately 1000 people and 35 nursing mothers with babies less than oneyear-old). Probability proportionate to size (PPS) sampling procedure was applied to determine proportionate sample size and number of clusters for each health area [12].

$$
\mathbf{n}_{\mathrm{o}}=f * N_{o}
$$

Where $n_{0}=$ health area sample size; $f=$ The sampling fraction $\left(n_{i} / N_{i}\right) ; N_{0}=$ The health area population; desired sample size $\left(n_{\mathrm{i}}\right)$ and the target population $\left(\mathrm{N}_{\mathrm{i}}\right)$. The sampling fraction $f$ is 0.1609 . The number of clusters and sample size of each health area was established (Table 1). In the cluster, all participants were sampled until the sample size was reached.

\section{Data Collection Tools}

An interviewer-administered structured questionnaire and a chart review (medical record review) form were used to collect relevant information from the participants.

The structured questionnaire was used to collect socio-demographic data and factors related to the knowledge of participants on IPTp-SP.

The chart review (medical record review) was used to collect information on the practice of IPTp-SP by participants. This information was reviewed from the ANC card of the participant.

\section{Study Procedures}

\section{Ethical approval and administrative authorisations}

After developing the research protocol, the investigator obtained ethical approval from the Faculty of
Health Sciences Institutional Review Board (FHSIRB), University of Buea. Administrative authorisations were then obtained from the Dean of the Faculty of Science, the Regional Delegate of Public Health for South West and the District Medical Officer (DMO) of Muyuka Health District.

\section{Pre-testing of data collection tools}

The investigator pre-tested the questionnaire and the chart review form on 20 participants in a community not involved in the study. This exercise helped to readjust some of the questions in order to increase validity of the data collection tools.

\section{Field data collection}

Participants for the study were selected from the 14 clusters in the study area. In all the clusters, the researcher went from house to house to enrol consenting participants who were present at the time of the visit until the sample size was reached. In the communities, the investigator together with community representatives identified the households with children age less than one year. The consent of participants was sought and those who gave their written consent were interviewed in a convenient location in the participant home. This process continued until the sample size was reached. A structured questionnaire (interviewer-administered), was used to collect socio-demographic data and knowledge of the participants on IPTp-SP. Chart review forms were used to collect information (secondary data) from the antenatal care (ANC) cards and delivery note of the participants. Self-reported uptake of SP was verified using the ANC card and was recorded.

\section{Data management}

Data collected were entered into an electronic questionnaire (template) created in Epi Info version 7 by the investigator. During the data entry process, $10 \%$ of data entered at the beginning was checked to ensure correct entry. The data were then cleaned or edited. The computer in which the data were stored was password protected and the information was accessible only to the researcher. The hard copies of the questionnaire were locked in a cupboard with the key accessible only to the researcher.

\section{Operational Definition of Terms}

\section{Intermittent Preventive Treatment of malaria in pregnancy (IPTp)}

Intermittent Preventive Treatment is the use of an-

Table 1: Proportionate samples for each health area.

\begin{tabular}{|l|l|l|l|}
\hline Health area & $\begin{array}{l}\text { Population of mothers with a child } \\
\text { less than one-year-old (a) }\end{array}$ & $\begin{array}{l}\text { Sample of mothers with a child less } \\
\text { than one-year-old (a*f) }\end{array}$ & $\begin{array}{l}\text { Number of clusters } \\
\text { selected (a*f)/30 }\end{array}$ \\
\hline Bafia & 712 & 114 & 4 \\
\hline Malende & 801 & 129 & 4 \\
\hline Muyuka & 1158 & 187 & 6 \\
\hline Total & 2671 & 430 & 14 \\
\hline
\end{tabular}


ti-malarial drugs given in treatment doses at predefined intervals after 16 weeks or after "quickening" to clear a presumed burden of placental malaria parasites. This is based on the assumption that malaria in pregnancy is asymptomatic in areas of stable malaria transmission.

\section{A full course of IPTp}

The administration of at least three doses of IPT during the pregnancy.

\section{A partial course of IPTp}

The administration of less than three doses of IPT during the pregnancy.

\section{Outcomes of pregnancy}

This was measured in terms of selected outcomes; birth weight, malaria in pregnancy and gestational age at delivery.

\section{Low birth weight}

Any birth weight lower than 2500 grams.

\section{Pre-term delivery}

Birth at gestation age earlier than 37 weeks.

\section{Clinical malaria in pregnancy}

Self-report of having been treated against clinical malaria during pregnancy. This was confirmed by verification from antenatal care (ANC) cards.

\section{Data Analysis}

Data collected were analysed using Epi info version 7. Participants' background characteristics data were analysed using means, standard deviation, frequency and proportion.

\section{To determine the adherence to a full course of IPTp- SP}

A univariate analysis was done using frequency and proportions.

\section{To determine the impact of adherence to full course of IPTp-SP on pregnancy outcome}

One-way analysis of variance (ANOVA) and multivariate binary logistic were used. Significant difference was established at $p$-value less than 0.05 .

\section{Ethical Considerations}

All participants included in the study had to give their written consent after receiving the information contained in the consent form. They signed the consent form in two copies and retained one. Those who were not able to read or write had to put their thumbprint on the form as signature after being sufficiently informed on the study. In this case, in addition, a legally acceptable representative of the participant who could read and write participated in the information session and
Table 2: Summary of socio-demographic characteristics of the participants.

\begin{tabular}{|c|c|c|}
\hline Characteristic & Frequency & Percentage (\%) \\
\hline Maternal age (years) & $\begin{array}{l}25.9 \pm 5.2 \\
\text { (Mean } \pm \text { SD) }\end{array}$ & 14-40 (Range) \\
\hline $14-20$ & 71 & 16.5 \\
\hline $21-30$ & 273 & 63.5 \\
\hline $31-40$ & 86 & 20 \\
\hline \multicolumn{3}{|l|}{ Marital status } \\
\hline Single/widow/divorced & 94 & 21.9 \\
\hline Married & 336 & 78.1 \\
\hline \multicolumn{3}{|l|}{ Health Area } \\
\hline Muyuka & 187 & 43.5 \\
\hline Bafia & 114 & 26.5 \\
\hline Malende & 129 & 30 \\
\hline \multicolumn{3}{|l|}{ Level of Education } \\
\hline No formal Education/Primary & 187 & 43.5 \\
\hline Secondary/University & 243 & 56.5 \\
\hline \multicolumn{3}{|l|}{ Occupation } \\
\hline Farmer/Student/No job & 283 & 65.8 \\
\hline Employed/Trader & 147 & 34.2 \\
\hline \multicolumn{3}{|l|}{ Religion } \\
\hline Christians & 425 & 98.8 \\
\hline Muslims & 5 & 1.2 \\
\hline \multicolumn{3}{|l|}{ Gravidity $^{*}$} \\
\hline Paucigravidae (G1/G2) & 268 & 62.3 \\
\hline Multigravidae (G3+) & 162 & 37.7 \\
\hline
\end{tabular}

*G1 = primigravidae; $\mathrm{G} 2$ = secundigravidae; G3+ = gravida 3 and above SD = Standard deviation.

also signed the consent form. They were given enough time to think, ask questions before taking their decision. For the cases of participants below 21-years-old, in addition to the consent that was obtained from the legal representative (parent or guardian), an assent was also obtained from the participant. Participation in the study was voluntary and there was no compensation. The participants were assured that they would be briefed on the research results. The risks related to the study were minimal. The time for the interview was between 10 to 15 minutes. The participants did not receive any penalty if they decided not to participate or to withdraw from the study at any time they wished to. The data from those who withdrew was not included in the analysis. Participants were assured of confidentiality and anonymity; their names did not figure on any of the data collection tools but contact numbers were collected for follow up if the need be.

\section{Results}

\section{Socio-demographic characteristics of participants}

The socio-demographic characteristics of the 430 participants included in this analysis are presented in Table 2. The mean age of the participants was 25.9 years and the range were $14-40$ years. Majority (63.5\%) of the participants were aged between 21-30 years-old. The sample population was just over three quarters (78\%) married/cohabiting women. The makeup of participants was $43.5 \%$ from Muyuka health area, $30 \%$ from 


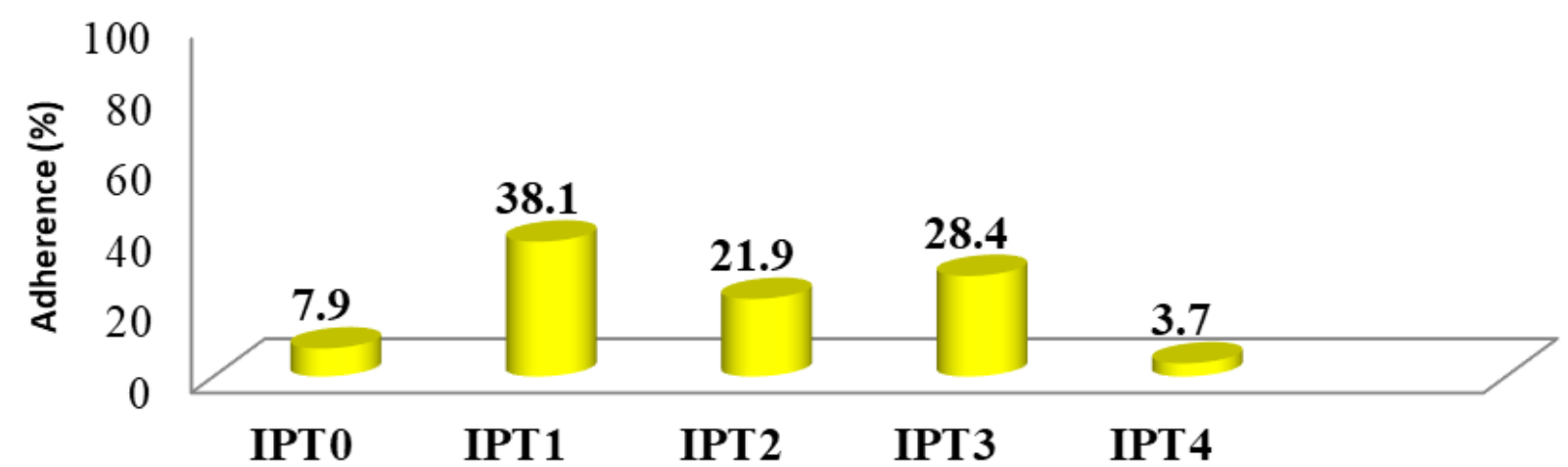

Figure 1: Adherence to IPT $, \mathrm{IPT}_{1}, \mathrm{IPT}_{2}, \mathrm{IPT}_{3}$ and $\mathrm{IPT}_{4}$.

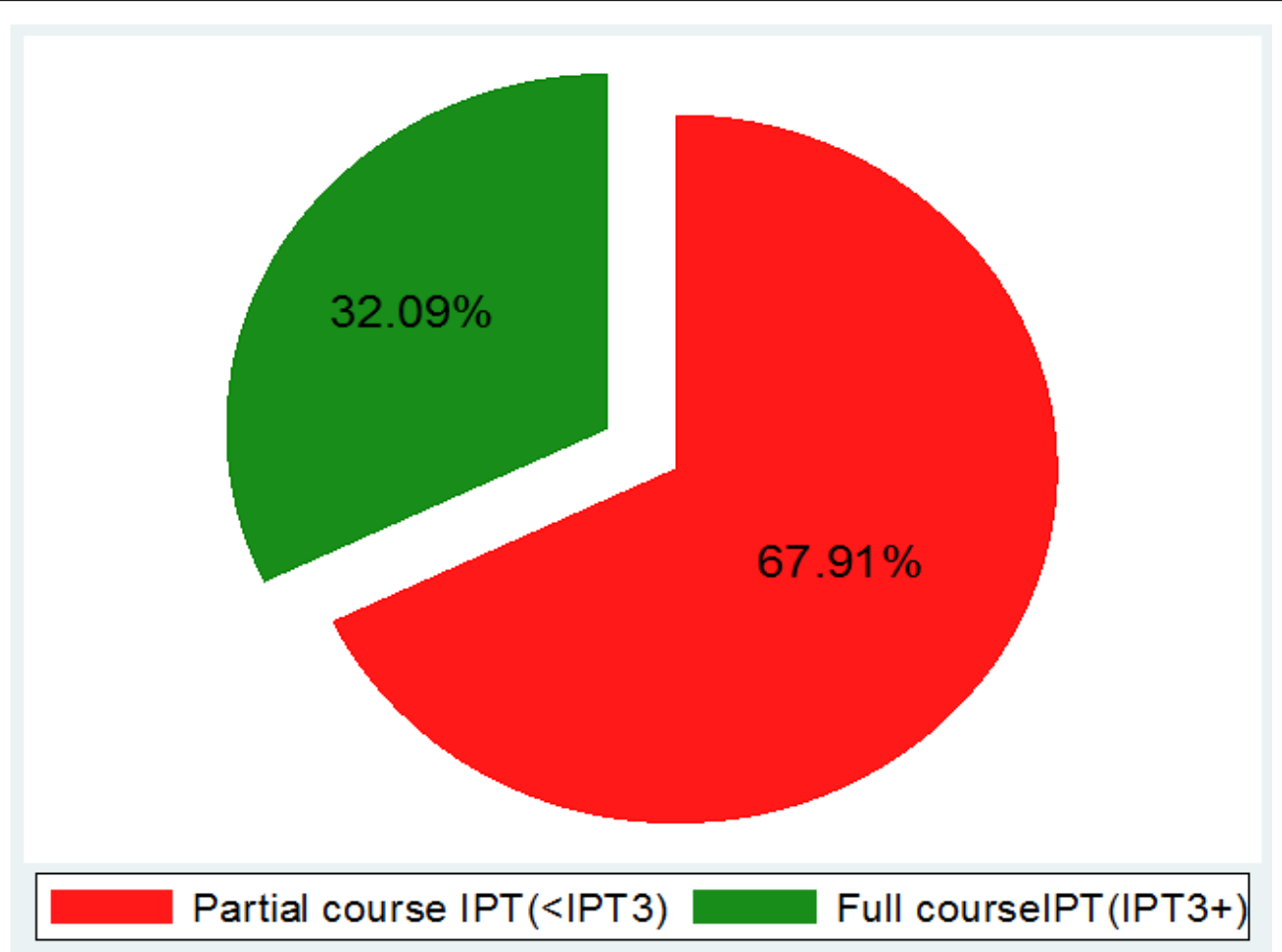

Figure 2: The prevalence of the uptake $\geq$ three doses SP among participants.

Table 3: Outcome of pregnancy among women who adhered and those who did not adhere to a full course of IPTp-SP.

\begin{tabular}{|l|l|l|l|}
\hline & Adhered to IPT & Did not adhere to IPT & p-value \\
\hline Birth weight $(\mathbf{k g})$ & $3.40 \pm 0.55$ & $3.14 \pm 0.58$ & $<0.001$ \\
\hline (Mean \pm SD) & & & \\
\hline Gestation age at delivery (weeks) & $38.02 \pm 2.2$ & $37.33 \pm 2.3$ & 0.003 \\
\hline (Mean \pm SD) & & & \\
\hline
\end{tabular}

Malende Health area and 26.5\% from Bafia health area. Just over half (56.5\%) of the participants had attended secondary school and beyond. The proportion of Pauci Gravidae women stood at $62.3 \%$. Very few $(1.2 \%)$ of the participants were Muslims by faith.

\section{Adherence to a full course of IPTp-SP}

Of the 430 participants, 34 (7.9\%), 164 (38.1\%), 94 (21.9\%), $122(28.4 \%)$ and $16(3.7 \%)$ reported taking none, one, two, three and four doses of SP during pregnancy respectively (Figure 1 ).

The proportion of participants who adhered to a full course ( $\geq$ three doses) of IPTp-SP was $32.09 \%$ (95\% Cl:

\section{7-36.8) as depicted in Figure 2.}

\section{Impact of adherence to full course of IPTp-SP on birth weight and gestation age at delivery}

Birth weight was significantly ( $p<0.001)$ higher in pregnant women who took a full course $(3.40 \pm 0.55)$ compared to those who took a partial course $(3.14 \pm$ 0.58). The difference in mean birth weight was $263 \mathrm{~g}$. Gestation age at delivery was significantly $(p=0.003)$ higher in pregnant women who took a full course (38.02 \pm 2.2 ) compared to those who took a partial course $(37.33 \pm 2.3)$ of IPTp (Table 3). The difference in mean gestation age at delivery was 0.69 weeks. 
Table 4: Secondary analysis of factors associated with Low birth weight (LBW) among participants.

\begin{tabular}{|c|c|c|c|c|}
\hline \multirow[t]{2}{*}{ Variable } & \multicolumn{2}{|l|}{ LBW (< $2500 \mathrm{~g})$} & \multirow[b]{2}{*}{ AOR (95\% Cl) } & \multirow[b]{2}{*}{ P-value } \\
\hline & COR (95\% CI) & P-value & & \\
\hline \multicolumn{5}{|l|}{ Maternal age } \\
\hline $14-20$ years & 1 & & 1 & \\
\hline $21-30$ years & $0.48(0.22-1.04)$ & 0.057 & $0.41(0.16-1.05)$ & 0.064 \\
\hline $31-40$ years & $0.60(0.30-1.45)$ & 0.07 & $0.59(0.25-1.50)$ & 0.075 \\
\hline \multicolumn{5}{|l|}{ Gravidity } \\
\hline Paucigravidae & 1 & & 1 & \\
\hline Multigravidae & $1.2(0.6-2.4)$ & 0.606 & $1.72(0.74-3.97)$ & 0.206 \\
\hline \multicolumn{5}{|l|}{ ANC visits } \\
\hline$<4$ visits & 1 & & 1 & \\
\hline$\geq 4$ visits & $0.15(0.06-0.37)$ & $<0.001$ & $0.22(0.07-0.71)$ & 0.012 \\
\hline \multicolumn{5}{|l|}{ SP doses taken } \\
\hline$<3$ doses & 1 & & 1 & \\
\hline$\geq 3$ doses & $0.24(0.08-0.7)$ & 0.005 & $0.82(0.2-3.3)$ & 0.775 \\
\hline \multicolumn{5}{|l|}{ Gestational age } \\
\hline Term delivery & 1 & & 11 & \\
\hline Pre-term delivery & $5.98(2.95-12.15)$ & $<0.001$ & $5.0(2.38-10.58)$ & $<0.001$ \\
\hline
\end{tabular}

ANC = Antenatal care; SP = Sulphadoxine-pyrimethamine; LBW = Low birth weight; COR = Crude odds ratio; $A O A=$ Adjusted odds ration; $95 \% \mathrm{Cl}=95 \%$ confidence interval; Significant $p$-values are presented in bold.

Table 5: Secondary analysis of factors associated with pre-term delivery among participants.

\begin{tabular}{|c|c|c|c|c|}
\hline Variable & Pre-term delivery & & & \\
\hline & COR $(95 \% \mathrm{CI})$ & P-value & AOR $(95 \% \mathrm{Cl})$ & P-value \\
\hline \multicolumn{5}{|l|}{ Maternal age } \\
\hline $14-20$ years & 1 & & 1 & \\
\hline $21-30$ years & $0.84(0.45-1.58)$ & 0.589 & $1.06(0.54-2.08)$ & 0.866 \\
\hline $31-40$ years & $0.90(0.52-1.70)$ & 0.324 & $1.20(0.65-2.20)$ & 0.412 \\
\hline \multicolumn{5}{|l|}{ Gravidity } \\
\hline Paucigravidae & 1 & & 1 & \\
\hline Multigravidae & $0.74(0.44-1.24)$ & 0.25 & $0.67(0.39-1.16)$ & 0.151 \\
\hline \multicolumn{5}{|l|}{ ANC visits } \\
\hline$<4$ visits & 1 & & 1 & \\
\hline$\geq 4$ visits & $0.40(0.24-0.67)$ & $<0.001$ & $0.29(0.14-1.16)$ & $<0.001$ \\
\hline \multicolumn{5}{|c|}{ SP doses taken } \\
\hline$<3$ doses & 1 & & 1 & \\
\hline$\leq 3$ doses & $0.69(0.40-1.20)$ & 0.187 & $1.63(0.75-3.50)$ & 0.215 \\
\hline
\end{tabular}

ANC = Antenatal care; SP = Sulphadoxine-pyrimethamine; PTD = Pre-term delivery $; 95 \% \mathrm{Cl}=95 \%$ confidence interval; Significant $p$-values are presented in bold.

\section{Impact of adherence to full course of IPTp-SP on Low Birth Weight}

The adherence to a full course IPTp-SP was not significantly associated with a reduction in the odds of low birth weight. The number of ANC visits and gestation age at delivery were significantly associated with a reduction in the odds of low birth weight. Attending more than 4 ANC visits conferred a $0.22(95 \% \mathrm{Cl}$ : 0.070.71 ) reduction in the odd of having an LBW compared to those who attended less than 4 ANC visits. Also, the odds of having an LBW in those who had a pre-term delivery was 5.0 times (95\% Cl: 2.38-10.58) that of those who had a term delivery (Table 4).

\section{Impact of adherence to full course of IPTp-SP on pre-term delivery}

The adherence to a full course IPTp-SP was not significantly associated with a reduction in the odds of preterm birth. The odds of having a pre-term birth in those who attended more than 4 ANC visits was 0.29 times ( $95 \% \mathrm{Cl}: 0.14-1.16)$ that of those who attended less than 4 ANC visits (Table 5).

\section{Impact of adherence to full course of IPTp-SP on malaria in pregnancy}

The uptake of $\geq$ three doses of SP (adherence) was associated with reduced odds of coming down with malaria in pregnancy $(A O R=0.30, p<0.001)$ compared to the uptake of $<$ three doses. Age and number of doses of SP significantly predicted the occurrence of malaria in pregnancy (Table 6). The effectiveness of IPTp-SP in preventing clinical malaria in pregnancy was 70\% [Effectiveness $=(1-$ odds ratio $) \times 100]$.

\section{Discussion}

\section{Adherence to a full course of IPTp-SP}

The proportion of participants who adhered to a full 
Table 6: Secondary analysis of factors associated with clinical malaria in pregnancy.

\begin{tabular}{|c|c|c|c|c|}
\hline \multirow[t]{2}{*}{ Variable } & \multicolumn{2}{|l|}{ Treated for MIP } & \multirow[b]{2}{*}{ AOR (95\% CI) } & \multirow[b]{2}{*}{ P-value } \\
\hline & COR $(95 \% \mathrm{CI})$ & P-value & & \\
\hline \multicolumn{5}{|l|}{ Maternal age } \\
\hline $14-20$ years & 1 & & 1 & \\
\hline $21-30$ years & $0.51(0.30-0.85)$ & 0.009 & $0.57(0.33-0.99)$ & 0.047 \\
\hline $31-40$ years & $0.40(0.20-0.64)$ & 0.005 & $0.45(0.25-0.70)$ & 0.03 \\
\hline \multicolumn{5}{|l|}{ Gravidity } \\
\hline Paucigravidae (G1/G2) & 1 & & 1 & \\
\hline Multigravidae ( $\geq$ G3) & $0.80(0.53-1.22)$ & 0.3 & $0.82(0.56-1.30)$ & 0.35 \\
\hline \multicolumn{5}{|l|}{ ANC visits } \\
\hline$<4$ visits & 1 & & 1 & \\
\hline$\geq 4$ visits & $0.64(0.43-0.96)$ & 0.031 & $1.23(0.76-2.03)$ & 0.398 \\
\hline \multicolumn{5}{|l|}{ SP doses taken } \\
\hline$<3$ doses & 1 & & 1 & \\
\hline$\geq 3$ doses & $0.33(0.19-0.53)$ & $<0.001$ & $0.30(0.17-0.54)$ & $<0.001$ \\
\hline
\end{tabular}

ANC = Antenatal care; SP = Sulphadoxine-pyrimethamine; MIP = Malaria in pregnancy; $95 \% \mathrm{Cl}=95 \%$ confidence interval; Significant $p$-values are presented in bold.

course of IPTp-SP was 32.1\%. This value is higher compared to household surveys carried out in other parts of Africa and Cameroon which revealed that the uptakes of full course of IPTp were $17 \%$ and $26 \%$ respectively $[2,9]$. This may reflect geographical variations in the coverage of IPTp-SP. The high prevalence of IPTp3+ observed in this study could be explained by the fact that most participants resided in areas accessible to health facilities providing ANC. Distance from health facility negatively correlates with the uptake of a full course of IPTp [13]. The last two years have seen the scaling up interventions towards the fight against maternal and child mortalities in Cameroon through the increase use of IPTp-SP interventions at the district levels.

However, the prevalence observed in our study is low compared to the $80 \%$ set as objective by the Cameroon Ministry of Public Health in its 2014-2018 strategic plans for the fight against Malaria [5]. This low uptake of a full of IPTp could be attributed to the low rate of ANC visits by participants. One hundred and ninety-eight participants (46\%) attended less than 4 ANC visits during pregnancy thus reducing the chances of taking a full course of IPTp. Our results were similar to those obtained in a study carried out in Ghana in a group of pregnant women where the prevalence of the adherence to a full course of IPTp-SP was 33\% [14].

\section{Impact of adherence to full course of IPTp-SP on outcomes of pregnancy}

Women who received a full course of IPTP-SP had better outcomes of their pregnancy. Low birth weight and prematurity are the greatest risk factors for neonatal mortality and a major contribution to infant mortality. In this study, babies born to mothers who received a full course of SP on the average weighed more than babies born to women who received a partial course of IPTp. They also had longer gestation age at delivery which reduced the risk of pre-term birth. This is similar to reports from a previous study in Ado-Ekiti, Nigeria where birth weight was significantly higher in PW who took SP compared to those who did not [15]. The high birth weight of babies in this study may not only be attributed to the low prevalence of malaria in pregnancy in them since other factors such as socio-economic level may also play a role.

The uptake of $\geq 3$ SP doses (adherence) was significantly associated with a $70 \%$ reduction in the prevalence of malaria in pregnancy. This demonstrates the efficacy of a full course of SP in improving the outcomes of pregnancy. This is comparable to reports from previous studies which indicated a reduction in the prevalence of malaria among women of all parities $[10,15,16]$. This supports the WHO recommendation to continue the use of SP as IPTp even in areas with high levels of SP resistance [2].

The adherence to three or more doses of SP was not associated with a significant reduction in the prevalence of LBW. The small sample size of the current study may not have provided the power needed to detect the effect on LBW. Infants born to women who attended ANC clinics four or more times were on average $156.7 \mathrm{~g}$ heavier than infants of women who had attended ANC clinics less than three times. This is similar to reports from a previous study carried out in Geita, Northwest of Tanzania which indicated a significant reduction in the prevalence of LBW in women who attended four or more ANC visits [9]. More ANC visits are associated with the receipt of more SP when readily available, women supplemented with haematinics, counseling on nutrition during pregnancy, all of which are associated with improved birth weight.

The adherence to full course of IPTp-SP was not significantly associated with reduced risk of pre-term delivery. However, women who attended more than 4 ANC visits (probably receiving the last dose of IPTp-SP within four weeks of delivery) had a $70 \%$ odds reduction of delivering pre-term infants. 


\section{Conclusions}

The adherence to of a full course of IPTp-SP was low compared to the national target of $80 \%$. The adherence to IPTp-SP showed significant geographical variations between health areas with Bafia health area having a significant lower adherence level.

The outcomes of pregnancy (birth weight, gestation age at pregnancy and malaria in pregnancy) were significantly better in women who took a full course of IPTp. Adherence to full course of IPTp-SP was significantly associated with reduced odds of coming down with malaria in pregnancy but not LBW and pre-term delivery.

\section{Recommendations}

Accelerate and scale up the implementation of the revised WHO policy on intermittent preventive treatment with sulphadoxine-pyrimethamine so as to improve on the adherence to a full course of IPTp-SP.

The continuous use of SP in preventing malaria in pregnancy bearing in mind that adherence improves pregnancy outcomes.

\section{Competing Interests}

The authors declare that they have no competing interests.

\section{Authors Contributions}

ATY conceptualized the problem, designed the study, performed data analysis and drafted the manuscript. TN and FJ supervised the work, were involved in designing the study, sorted clinical issues around the problem, and reviewed the manuscript critically. NEN, CTD, FSW and KEN collected and analysed the data. All authors read and approved the final draft of the manuscript.

\section{Acknowledgement}

I sincerely thank my supervisors Prof. Theresa Nkuo-Akenji and Dr. Fru-Cho Jerome for guiding me throughout this research work. I am very grateful for the sacrifices they made and the patience they exercised throughout the course of this work. My gratitude to the University of Buea for the facilities placed at my disposal to enable my studies. I immensely thank the Head of Department of Microbiology and Parasitology-Prof. Wanji Samuel and the other staff of the Department for imparting knowledge to us. I am also very grateful to the Regional Delegate of Public Health for the South West Region- Dr. Mbome Victor Njie and to the District Medical Officer for Muyuka Health District-Dr. Mboum Alphones for the administrative authorisations to collect the research data in the Muyuka Health District. I thank all the nursing mothers in the Muyuka Health District who participated in this research work. I also appreciate the collaboration and mutual support of my classmates during the course of this program. Special thanks to Kah Emmanuel and Njukang Ernest for their contribution and encouragement. Above all I wish to thank the Almighty God for his abundant grace in my life.

\section{References}

1. Greenwood BM, Bojang K, Whitty CM, Target GA (2005) Malaria. Lancet 365: 1487-1498.

2. (2014) WHO: World Malaria Report. Geneva.

3. Guyatt HL, Snow RW (2001) Malaria in pregnancy as an indirect cause of infant mortality in sub-Saharan Africa. Trans R Soc Trop Med Hyg 95: 569-576.

4. Aribodor DN, Nwaorgu OC, Eneanya Cl, Okoli I, Etaga HO, et al. (2009) Association of low birth weight and placental malarial infection in Nigeria. J Infect Dev Ctries 3: 620-623.

5. (2012) NMCP: 2012 Annual Report. Yaounde.

6. (2004) WHO: A strategic framework for malaria prevention and control during pregnancy in African Region. Brazzaville. World Health Organisation.

7. Tchinda VH, Socpa V, Keundo AA, Zeukeng F, Seumen CT, et al. (2012) Factors associated to bed net use in Cameroon: A retrospective study in Mfou health district in the centre region. Pan Afr Med J 12: 112.

8. (2014) WHO: WHO policy brief for the implementation of intermittent preventive treatment of malaria in pregnancy using sulphadoxine-pyrimethamine (IPTp-SP) April 2013(revised January 2014).

9. (2015) DHS-MICS: 2014 preliminary Report. Cameroon.

10. Mpogoro FJ, Matovelo D, Dosani A, Ngallaba S, Mugono $M$, et al. (2014) Uptake of intermittent preventive treatment with sulphadoxine-pyrimethamine for malaria during pregnancy and pregnancy outcomes: A cross-sectional study in Geita district, North-Western Tanzania. Malar J 13: 455.

11. Naing L, Winn T, Rusli B (2006) Practical issues in calculating the sample size for prevalence studies. Archives of Orofacial Science 1: 9-14

12. Chepkemoi NM (2013) Factors influencing the uptake of intermittent preventive treatment for malaria in pregnancy: Evidence from Bungoma East District, Kenya. American Journal of Public Health Research 1: 110-123.

13. Exavery A, Mbaruku G, Mbuyita S, Makemba A, Kinyonge IP, et al. (2014) Factors affecting uptake of optimal doses of sulphadoxine-pyrimethamine for intermittent preventive treatment of malaria in pregnancy in six districts of Tanzania. Malar J 13: 22.

14. Tutu EO, Lawson B, Browne E (2011) The effectiveness and perception of the use of sulphadoxine-pyrimethamine in intermittent preventive treatment of malaria in pregnancy programme in Offinso district of Ashanti region, Ghana. Malarial Journal 10: 385.

15. Aduloju O (2013) Effect of intermittent preventive treatment of malaria on the outcome of pregnancy among women attending antenatal clinic of a new Nigerian teaching hospital, Ado-Ekiti. Niger Med J 54: 170-175.

16. Onyebuchi AK, Onoh CH, lyoke CA, Lawani LO, Okeke NE (2014) Adherence to intermittent preventive treatment for malaria with sulphadoxine-pyrimethamine and outcome of pregnancy among parturients in South east Nigeria. Dove Press 8: 447-452.
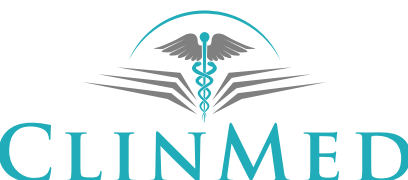

INTERNATIONAL LIBRARY 